\title{
An open pilot study of zonisamide augmentation in major depressive patients not responding to a low dose trial with duloxetine: preliminary results on tolerability and clinical effects
}

Michele Fornaro ${ }^{1}$, Matteo Martino ${ }^{1 *}$, Bruna Dalmasso ${ }^{2}$, Salvatore Colicchio ${ }^{3}$, Marzia Benvenuti ${ }^{4}$, Giulio Rocchi ${ }^{1}$, Andrea Escelsior ${ }^{1}$ and Giulio Perugi ${ }^{4}$

\begin{abstract}
Background: Despite multiple antidepressant options, major depressive disorder (MDD) still faces high nonresponse rates, eventually requiring anticonvulsant augmentation strategies too. The aim of this study was to explore such a potential role for zonisamide.

Methods: A total of 40 MDD outpatients diagnosed using the Diagnostic and Statistical Manual for Mental Disorders, fourth edition criteria entered a 24 week open trial receiving duloxetine $60 \mathrm{mg} /$ day for the first 12 weeks and subsequently (weeks 12 to 24) augmentation with zonisamide $75 \mathrm{mg} /$ day if they did not respond to the initial monotherapy. Efficacy and tolerability were assessed using the Hamilton Scales for Anxiety and Depression (a 12 week score $\geq 50 \%$ vs baseline defined 'non-response'), the Arizona Sexual Experience Scale, the Patient Rated Inventory of Side Effects and the Young Mania Rating Scale.

Results: At week 12, 15 patients out of 39 (38.5\%) were responders, and 1 had dropped out; remarkably, 14 patients out of $24(58.3 \%)$ had achieved response by week 24. Poor concentration and general malaise were associated with non-response both at week 12 and $24(P=0.001)$, while loss of libido and reduced energy were prominent among final timepoint non-responders. Patients receiving zonisamide also experienced weight reduction $(2.09 \pm 12.14 \mathrm{~kg} ; \mathrm{P}=0.001)$ independently of the outcome.

Conclusions: Although only a preliminary study due to strong methodological limitations, and thus requiring confirmation by further controlled investigations, the current results indicate zonisamide may be a potential augmentation option for some depressed patients receiving low doses of duloxetine.
\end{abstract}

\section{Introduction}

Major depressive disorder (MDD) is significant cause of morbidity and mortality, and is associated with a high socioeconomic burden [1]. Both pharmacological and non-pharmacological interventions have proven efficacy in many MDD cases, yet failure to respond to standard interventions still represents a frequent scenario [2]. Among other issues, nosological boundaries and pharmacological issues might lead to unfavorable outcomes,

\footnotetext{
* Correspondence: matteomartino9@libero.it

'Department of Neuroscience, Section of Psychiatry, University of Genova, Genoa, Italy

Full list of author information is available at the end of the article
}

prompting pharmacological augmentation strategies [3] even for 'proven effective' antidepressants such as the serotonergic norepinephrinergic reuptake inhibitors (SNRIs) [4].

A pharmacological augmentation strategy involves a number of agents from different classes, including some anticonvulsants routinely prescribed in the clinical setting both for anxious states [5] and/or depression [6], sometimes controversially $[7,8]$ or even in the absence of a bipolar course of illness [6].

To mention one, lamotrigine is an anticonvulsant drug often used as an antidepressant augmentation therapy even for non-bipolar patients, although its use as

\section{C) Biomed Central}


augmentation strategy for treatment-resistant unipolar depression is supported by only a single randomized clinical trial [9]. While it may be argued that many Diagnostic and Statistical Manual for Mental Disorders, fourth edition (DSM-IV) [10] cases of depression should indeed follow a bipolar diathesis, suggesting prudent (or low dose) prescription of antidepressants and/or augmentation therapies with antimanic agents [2], such a prescribing habit is a popular clinical practice also supported by pharmacodynamic considerations. With regard to lamotrigine, its actions include blockade of sodium and calcium channels, hypothetically leading to reduced $N$-methyl-D-aspartate glutamatergic transmission as well as changes in the activity of crucial neurotransmitters involved in the pathophysiology of depression, including dopamine and serotonin [11-13]. Therefore, in consideration of a partial pharmacodynamic overlap between lamotrigine and the latterly introduced zonisamide [14-16] (at least with regard to a common putative modulation of glutamate and monoamines), and accounting for the drug concentrationrelated biphasic effects of zonisamide on serotonergic system functioning in rat hippocampus [15], zonisamide should also receive attention for its potential role in the management of some psychiatric disturbances, as recently proposed for anxiety disorders refractory to standard anxiolytic medications [17]. Additionally, zonisamide (a sulfonate anticonvulsant drug with long half life (65 h in plasma) approved for use as an adjunctive therapy in adults with partial-onset seizures, infantile spasm, mixed seizure types of Lennox-Gastaut syndrome, myoclonic, and generalized tonic clonic seizure), when added at 25 to $50 \mathrm{mg} /$ day to commonly used anti-Parkinsonian drugs, significantly improved the primary symptoms of patients with advanced Parkinson's disease, possibly by activation of dopamine synthesis, inhibition of monoamine oxidase type $\mathrm{B}$, inhibition of T-type calcium channels and inhibition of an indirect pathway in the basal ganglia through the sigma opioid receptor [18]. Therefore, zonisamide's propensity to facilitate dopaminergic and serotonergic release in vivo [19] might suggest an exploration of its potential role as augmentation strategy for common antidepressant drugs is prudent, possibly even at dosages lower than the ones usually adopted for the treatment of epileptic conditions.

Therefore, in this study we explore the efficacy and tolerability of adjunctive zonisamide in the treatment of MDD not responsive to a preliminary trial of the SNRI antidepressant duloxetine administered at low dose (60 $\mathrm{mg} /$ day).

\section{Methods}

\section{Study design}

This was a preliminary 24 week open trial designed to assess the efficacy and tolerability of zonisamide $75 \mathrm{mg}$ / day augmentation for MDD patients (actually an offlabel prescription of zonisamide) not responding to a 12 week treatment with duloxetine at $60 \mathrm{mg} /$ day. The unusual choice of duloxetine was essentially dictated by pharmacokinetic and pharmacodynamic issues, in view of subsequent combination with zonisamide. The study was conducted from February 2008 to September 2010, with approval by the Ethical Committee of the San Martino Hospital, University of Genova, Genoa, Italy in November 2007.

\section{Subjects}

The planned and actual study population included 40 outpatients, aged 18 years or older, of both genders, fulfilling DSM-IV criteria for MDD and with a current single or recurrent major depressive episode. At screening, patients had to have a minimum score of 18 on the 17item Hamilton Scale for Depression (HAM-D) [20]. Exclusion criteria included the following DSM-IVdefined diagnoses: bipolar disorders (either type I or type II), cyclothymia, schizoaffective disorder or schizophrenia, dementia or substance abuse disorder in the last 6 months, suicidal ideation that made participation unduly risky, unstable medical conditions, abnormal thyroid function, QTc $\geq 450 \mathrm{~ms}$ on screening electrocardiogram (ECG; calculated using the Bazett formula), being pregnant, lactating, or not using adequate contraception if capable of getting pregnant, as well as having known contraindications for zonisamide (for example, history of severe myopia, kidney stones or narrow angle glaucoma) or duloxetine. Patients were also excluded if unwilling or unable to provide valid signed informed consent or if they could not safely taper concomitant psychotropic drugs, which had to be withdrawn for 2 weeks, except for fluoxetine and depot neuroleptics requiring at least a 4 week discontinuation. Zolpidem 10 $\mathrm{mg}$ at bedtime was allowed, but could not be taken the night before scheduled assessments. Finally, body weight (in $\mathrm{kg}$ ) was also recorded at screening, week 12 (main evaluation time) and week 24 (endpoint) along with repeated medical monitoring including ECG recording. Patients could leave the study at any time and still obtain clinical care.

\section{Study procedures and efficacy measures}

Diagnoses were made by clinical examination and the Structured Clinical Interview for DSM-IV Axis-I Disorders/Patient Edition (SCID-I/P) [21]. At baseline, eligible patients started taking duloxetine $60 \mathrm{mg} /$ day once a day after being evaluated by means of the HAM-D, Hamilton Scale for Anxiety (HAM-A) [22], Young Mania Rating Scale (YMRS) [23] and the Arizona Sexual Experience Scale (ASEX) [24]. All measurements were repeated at week 12 and week 24 while the Patient 
Rated Inventory of Side Effects (PRISE) [25] was administered at week 12 and week 24 as primary tolerability evaluation. As major outcome measurement, a week 12 HAM-D total score $\leq 50 \%$ vs baseline defined 'nonresponse'. Similarly, an endpoint HAM-D total score $\leq 50 \%$ vs baseline was adopted to define (final) 'responders' (primary endpoint) or 'remission' if $<7$.

\section{Data analysis}

Both descriptive and analytical analyses $\left(\chi^{2}\right.$ or $t$ tests when appropriate) were performed using SPSS V.19 for Windows (SPSS, Chicago, IL, USA). Two-tailed tests with a $5 \%$ level of significance were used through the analyses. Since the data followed a normal distribution (assessed by Kolmogorov-Smirnov test), only parametric analyses were conducted. Finally, as a result of the very low number of dropout cases (in fact, $\mathrm{n}=1$; see below) an intent to treat analysis was performed.

\section{Results}

A total of 40 patients, all of Caucasian origin, constituted the study sample. Two patients dropped out before week 12 and week 24, respectively. The first dropout case did not attend the scheduled follow-up, giving no reason, while the second did not complete the final follow-up for (clinically confirmed) depressed mood: although not fulfilling the HAM-D scale at week 24 , this subject was included in the 'non-responders' group, being therefore considered in the final statistical analysis. The mean HAM-D reduction for the group as a whole from baseline (20.53) to week 12 (10.08) was -10.45 . At screening, mean age was $(47 \pm 10.7), F=24$ (60\%) and $M=16$ (40\%); none of the patients had relevant medical or psychiatric Axis-I comorbidities and $84 \%$ of the sample experienced first (considered as single) major depressive episode, with a mean baseline HAM-D score $=23$ (18 to 24 scores indicate moderate/ average depression). At week 12, 15 out of 39 (38.5\%) subjects were 'responders' while 24 (61\%) were not, thus continuing the study with zonisamide $75 \mathrm{mg} /$ day once a day augmentation and maintaining duloxetine $60 \mathrm{mg} /$ day once a day. By definition, week 12 HAM-D total scores were significantly lower among responders $(P=$ $0.001)$ as this was the case of HAM-A total scores $(P=$ $0.001)$, Figure 1.

With regard to the side effects profile, 'anorgasmy' ( $P=0.003)$, 'poor concentration' and 'general malaise' (both $P=0.001$ ) were more frequent among preliminary non-responders vs responders, as shown in Table 1. At week 24, the general sexual (side effect)

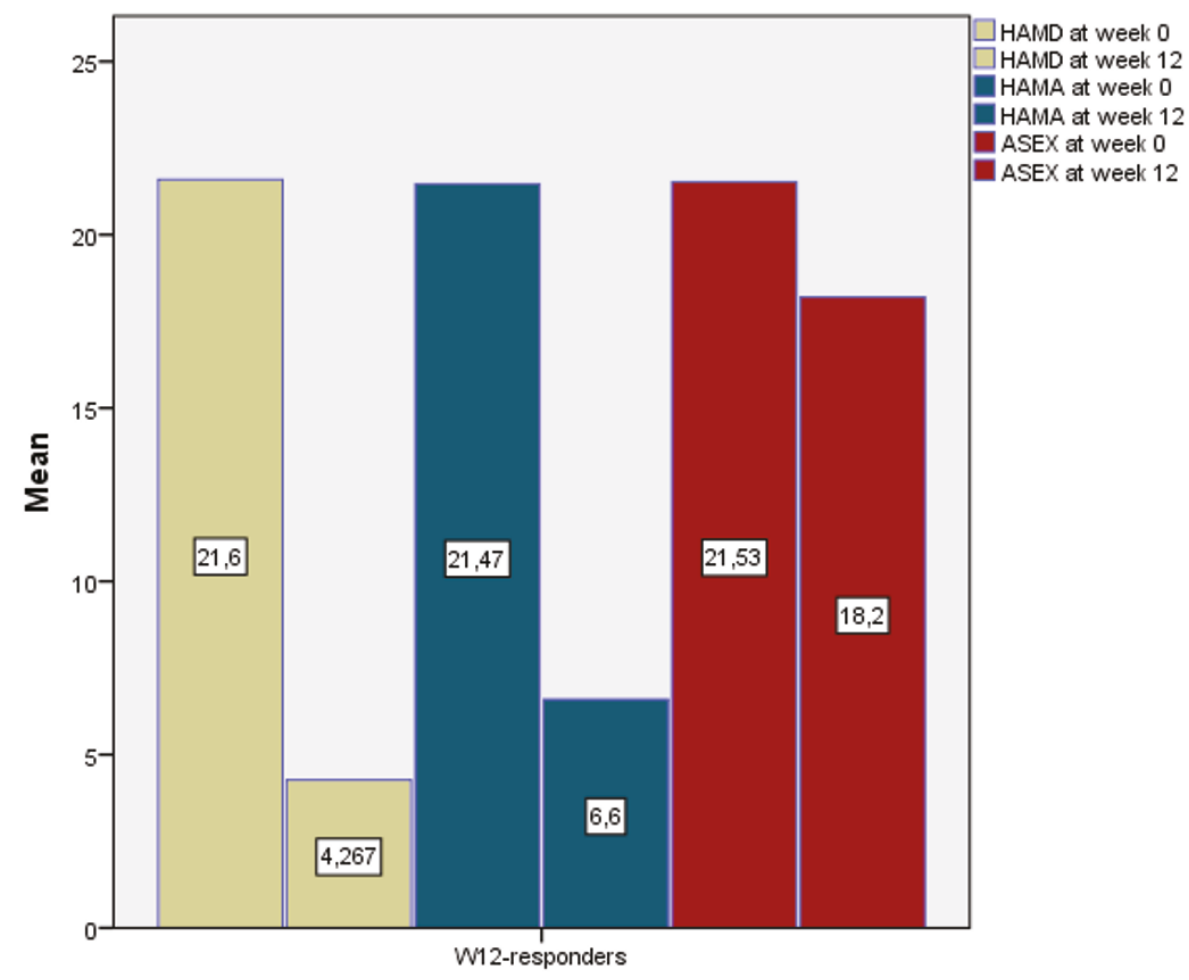

Figure 1 Mean values for HAM-D, HAM-A and ASEX at baseline and week 12 in responder patients concluding the trial prior to receiving zonisamide augmentation. ASEX = Arizona Sexual Experience Scale; HAM-A = Hamilton Anxiety Scale; HAM-D = Hamilton Depression Scale; YMRS = Young Mania Rating Scale. 
Table 1 Demographic and clinical features week 12 comparison of responders vs non-responders

\begin{tabular}{|c|c|c|c|c|}
\hline & Responders, $\mathrm{N}=15$ (38.5\%) & Non-responders, $\mathrm{N}=24$ (61.5\%) & $\mathrm{t}$ or $\chi^{2}(\mathrm{df}=1)$ & $P$ value \\
\hline Age, mean(s) & $41.33 \pm 9.5$ & $50.7 \pm 0.2$ & 2.865 & NS \\
\hline General features & $F=9 ; M=6$ & $F=1 ; M=23$ & -0.152 & NS \\
\hline Weight (in kg) & $68.4 \pm 15.71$ & $68.29 \pm 12.11$ & 36 & NS \\
\hline \multicolumn{5}{|l|}{ Clinical features (\%) } \\
\hline HAM-D total score & $4.27 \pm 3.15$ & $13.71 \pm 2.53$ & 10.320 & 0.001 \\
\hline HAM-A total score & $6.60 \pm 3.58$ & $12.83 \pm 4.76$ & 4.351 & 0.001 \\
\hline YMRS total score & $1.93 \pm 1.62$ & $3.21 \pm 1.16$ & 2.394 & 0.022 \\
\hline ASEX total score & $18.20 \pm 5.69$ & $18.58 \pm 4.87$ & 0.224 & NS \\
\hline \multicolumn{5}{|l|}{ Side effects profile (\%) } \\
\hline$\underline{\text { Diarrhea }}$ & 0 & $4(10.25 \%)$ & 2.786 & NS \\
\hline Tremors & 0 & $2(5.12 \%)$ & 1.318 & NS \\
\hline Poor coordination & 0 & $2(5.12 \%)$ & 1.318 & NS \\
\hline Dizziness & 0 & $4(10.25 \%)$ & 2.786 & NS \\
\hline$\underline{\text { Blurred vision }}$ & $3(7.7 \%)$ & $6(15.4 \%)$ & 0.130 & NS \\
\hline Ringing in ears & $4(10.25 \%)$ & 0 & 7.131 & 0.008 \\
\hline Difficult urination & $4(10.25 \%)$ & $2(5.12 \%)$ & 2.383 & NS \\
\hline Painful urination & 0 & $2(5.12 \%)$ & 1.318 & NS \\
\hline Pollachiuria & $1(2.6 \%)$ & $2(5.12 \%)$ & 0.036 & NS \\
\hline Menstrual irregularity & 0 & 0 & - & - \\
\hline Insomnia & $11(28.2 \%)$ & $18(46.15 \%)$ & 0.013 & NS \\
\hline Hypersomnia & 0 & $4(10.25 \%)$ & 2.786 & NS \\
\hline Loss of libido & $4(10.25 \%)$ & $12(30.77 \%)$ & 2.077 & NS \\
\hline Anorgasmy & $4(10.25 \%)$ & $18(46.15 \%)$ & 8.770 & 0.003 \\
\hline Erectile dysfunction & 0 & 0 & - & - \\
\hline Poor concentration & $1(2.6 \%)$ & $16(41 \%)$ & 13.514 & 0.001 \\
\hline General malaise & 0 & $14(35.9 \%)$ & 13.650 & 0.001 \\
\hline Reduced energy & $3(7.7 \%)$ & $12(30.77 \%)$ & 3.510 & NS \\
\hline Hyperphagia & $2(5.12 \%)$ & $4(10.25 \%)$ & 0.079 & NS \\
\hline
\end{tabular}

ASEX = Arizona Sexual Experience Scale; HAM-A = Hamilton Anxiety Scale; HAM-D = Hamilton Depression Scale; NS = not significant; YMRS = Young Mania Rating Scale.

profile (ASEX total) was poorer in final non-responders $(\mathrm{n}=10,41.7 \%)$, with 'loss of libido' and 'anorgasmy' (both $P=0.001$ ) among the most frequent complaints; 'general malaise' and 'poor concentration' remained prevalent among non-responders (both $P=$ $0.001)$, associated with 'reduced energy' $(P=0.001)$ compared to final responders $(\mathrm{n}=14,58.3 \%)$, as reported in Figure 2 and Table 2. No patients evolved to a manic episode (defined by YMRS total score $\geq 13$ ) or developed clinically relevant medical adverse events during the follow-up period. Remarkably, patients treated with zonisamide experienced significant weight reduction (mean $2.09 \pm 12.14 \mathrm{~kg} ; P=$ 0.001 ) independently of their final outcome (mean $2.79 \pm 11.67 \mathrm{~kg}$ and $1.39 \pm 12.61 \mathrm{~kg}$ in responders and non-responders, respectively), as shown in Figure 3 , whereas mean week 12 weight did not statistically differ from baseline values.

\section{Discussion}

By the end of the study, 29 (72.5\%) out 40 patients had achieved response $(51.7 \%$ with duloxetine monotherapy and $48.3 \%$ with both duloxetine and zonisamide). Nonetheless, a number of issues must be raised prior to considering the findings from the present pilot study. Primarily, this was a small sample, low-powered, open trial, thus its validity is limited by the absence of a control (and regression analysis techniques). Also, while the sample appeared quite homogeneous and prone to good compliance toward medications, it included mainly first (possibly single) episode major depressed patients with mainly mild to moderate cases of depression (as indicated by respective baseline HAM-D scores), thus making the study prone to a Berkson bias ('exclusion of most severe cases leading to potential distortion of statistical results'). Moreover, stating the explorative nature of this pilot study, we used low doses of drugs 


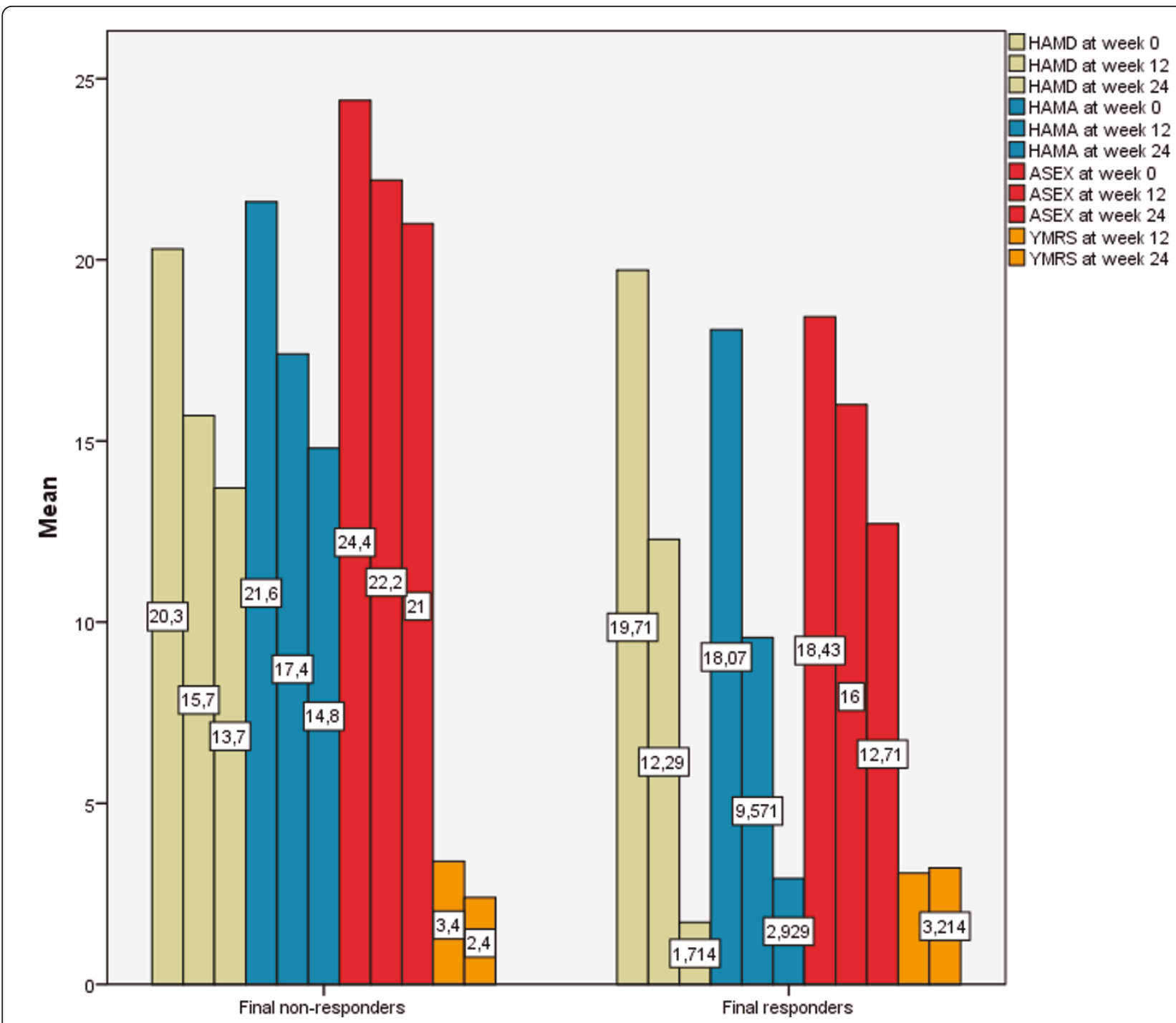

Figure 2 Trends of HAM-D, HAM-A, ASEX and YMRS from baseline to week 12 and to week 24 in final responders and final nonresponders. ASEX = Arizona Sexual Experience Scale; HAM-A = Hamilton Anxiety Scale; HAM-D = Hamilton Depression Scale; YMRS = Young Mania Rating Scale.

essentially for safety considerations. Hypothetically, some of the patients not responding at week 12 on duloxetine fixed-dose monotherapy may have responded if treated with higher doses of antidepressant (for example, $120 \mathrm{mg} /$ day) and/or if treated for longer, although when an antidepressant response is observed it usually begins within the first months of treatment [26]. They may have also responded to an eventual placebo or even spontaneously due to the natural course of MDD. In this sense, it cannot be determined if and how any of the patients receiving zonisamide represented a true 'treatment resistant depression' case.

While this remains a major constraint of this pilot study, the use of low dosages of zonisamide (compared to anticonvulsant ranges) was essentially due to the explorative nature of the investigation and absence of specific guidance for its use for MDD. However, it should be considered that zonisamide is commonly used at dosages between 25 to $50 \mathrm{mg} /$ day as augmentation therapy for common anti-Parkinsonian drugs. Moreover, while the concomitant use of duloxetine and zonisamide for the last 12 weeks of the study should be seen as a further confounding factor in discriminating the therapeutic effect of each single agent, this pilot study rather aimed to investigate the role of the combination of the two drugs, and the unusual choice of the SNRI duloxetine (mainly metabolized by CYP1A2 and CYP2D6) was essentially due to its low propensity for pharmacokinetic interactions with zonisamide (mainly metabolized via CYP3A4). 
Table 2 Week 24 comparison of demographic and clinical features of responders vs non-responders

\begin{tabular}{|c|c|c|c|c|}
\hline & Responders, $\mathrm{N}=14$ (58.3\%) & Non-responders, $\mathrm{N}=10(41.7 \%)$ & $\mathrm{t}$ or $\chi^{2}(\mathrm{df}=1)$ & $P$ value \\
\hline Weight (in kg) & $65.5 \pm 11.23$ & $65.90 \pm 13.12$ & 22 & NS \\
\hline \multicolumn{5}{|l|}{ Clinical features (\%) } \\
\hline HAM-D total score & $1.71 \pm 1.32$ & $13.70 \pm 5.14$ & 8.405 & 0.001 \\
\hline HAM-A total score & $2.93 \pm 2.05$ & $14.80 \pm 4.21$ & 9.182 & 0.001 \\
\hline YMRS total score & $3.21 \pm 5.76$ & $2.40 \pm .843$ & -0.440 & NS \\
\hline ASEX total score & $12.71 \pm 1.89$ & $18.58 \pm 4.87$ & 5.129 & 0.001 \\
\hline \multicolumn{5}{|l|}{ Side effects profile (\%) } \\
\hline Diarrhea & 0 & 0 & - & - \\
\hline Tremors & 0 & 0 & - & - \\
\hline Poor coordination & 0 & $2(8.3 \%)$ & 3.055 & NS \\
\hline Dizziness & 0 & 0 & - & - \\
\hline Blurred vision & 0 & 0 & - & - \\
\hline Ringing in ears & 0 & 0 & - & - \\
\hline Difficult urination & 0 & $4(16.7 \%)$ & 6.720 & 0.010 \\
\hline Painful urination & 0 & 0 & - & - \\
\hline Pollachiuria & 0 & $4(16.7 \%)$ & 6.720 & 0.010 \\
\hline Menstrual irregularity & 0 & 0 & - & - \\
\hline Insomnia & $2(8.3 \%)$ & $4(16.7 \%)$ & 2.057 & NS \\
\hline Hypersomnia & 0 & $2(8.3 \%)$ & 3.055 & NS \\
\hline Loss of libido & 0 & $8(33.3 \%)$ & 16.800 & 0.001 \\
\hline Anorgasmy & 0 & $6(25 \%)$ & 11.200 & 0.001 \\
\hline Erectile dysfunction & 0 & 0 & - & - \\
\hline Poor concentration & 0 & $10(41.7 \%)$ & 24.000 & 0.001 \\
\hline General malaise & 0 & $6(25 \%)$ & 11.200 & 0.001 \\
\hline Reduced energy & 0 & $10(41.7 \%)$ & 24.000 & 0.001 \\
\hline Hyperphagia & 0 & 0 & - & - \\
\hline \multicolumn{5}{|c|}{$\begin{array}{l}\text { 'Anorgasmy', 'poor concentration' and 'general malaise' were already significantly improved in those patients responding to a } 12 \text { week trial with duloxetine } \\
\text { monotherapy and appear to still be associated to response even at week } 24 \text { among those patients who continued the study. Also, the difference in the ASEX } \\
\text { scores between responders and non-responders at the end of the study is likely due to the fact that the non-responders' scores substantially did not modify } \\
\text { within week } 12 \text { (before zonisamide augmentation) and week } 24 \text {, while it reduced in final responders. }\end{array}$} \\
\hline \multicolumn{5}{|c|}{$\begin{array}{l}\text { ASEX = Arizona Sexual Experience Scale; HAM-A = Hamilton Anxiety Scale; HAM-D = Hamilton Depression Scale; NS = not significant; YMRS = Young Mania } \\
\text { Rating Scale. }\end{array}$} \\
\hline
\end{tabular}

Finally, the present findings and hypotheses must be considered as merely speculative due to the shortcomings listed above. The present investigation was a small open-label uncontrolled study, therefore no firm conclusions can be drawn from the results; it should be considered as a pilot study for further rigorous investigation, essentially prompted by the fact that zonisamide appeared to be a well tolerated augmentation therapy (however, the absence of placebo control might be somehow misleading), with low dropout rates even in the presence of some side effects (although it should be remembered that people who recover from depression, for whatever reason, are also less likely to endorse a list of somatic complaints and that some other complaints, including sexual ones, could be part of MDD rather than side effects due to treatment). What should be noted is that zonisamide apparently did not produce negative effects compared with the start of treatment and that, since weight gain is a common complaint among MDD patients receiving standard antidepressants and a major potential cause of drug withdrawal, the observation of weight reduction in the presence of zonisamide augmentation added to a low-dose duloxetine suggests further methodologically rigorous, controlled studies would be warranted.

\section{Author details}

${ }^{1}$ Department of Neuroscience, Section of Psychiatry, University of Genova, Genoa, Italy. ${ }^{2}$ Department of Hematology and Oncology, Section of Semeiotics and Medical Methodology I, University of Genova, Genoa, Italy. ${ }^{3}$ Department of Neurosciences, Catholic University of Rome, Rome, Italy. ${ }^{4}$ Department of Psychiatry, University of Pisa, Pisa, Italy.

\section{Authors' contributions}

MF conceived the study and performed the statistical analysis, MM contributed to manuscript drafting and BD performed the physical 


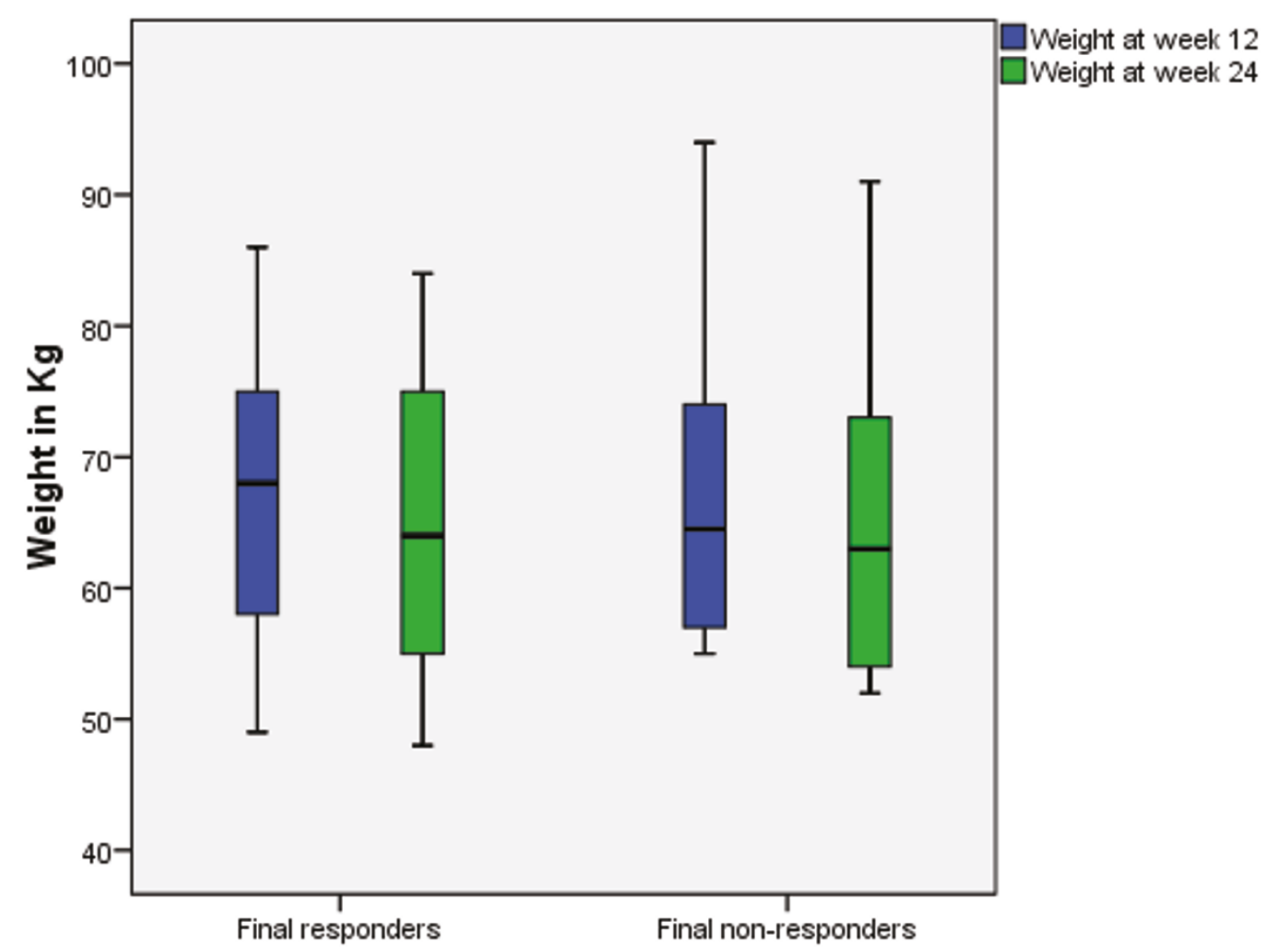

Figure 3 Comparison of mean weight (in $\mathbf{k g}$ ) at weeks 12 and 24 in final responders vs final responders. Those patients receiving zonisamide experienced a slight, yet statistically significant $(P=0.001$ both) weight reduction independently of the outcome. ASEX $=$ Arizona Sexual Experience Scale; HAM-A = Hamilton Anxiety Scale; HAM-D = Hamilton Depression Scale; YMRS = Young Mania Rating Scale.

examinations. MB, GR, AE and SC helped in retrieving literature references and/or in patient enrollment and follow-up. GP served as senior study consultant. All authors read and approved the final version of the manuscript.

\section{Competing interests}

The authors declare that they have no competing interests, including any connection to Eisai or Elan.

Received: 5 June 2011 Accepted: 19 September 2011 Published: 19 September 2011

\section{References}

1. Tylee A, Walters P: The burden of depression. Hosp Med 2002, 63:580-581.

2. Fornaro $M$, Giosue P: Current nosology of treatment resistant depression: a controversy resistant to revision. Clin Pract Epidemiol Ment Health 2010, 6:20-24.

3. Fava M: Augmentation and combination strategies in treatment-resistant depression. J Clin Psychiatry 2001, 62(Suppl 18):4-11.

4. Petersen T, Perlis RH, Ticknor C, Lohr J, Solvason HB, O'Reardon JP, Wohlreich MM, Andreotti C, Wilson M, Fava M: Efficacy of duloxetine for the treatment of depression: relationship to most recent antidepressant trial. Psychopharmacol Bull 2008, 41:34-45.

5. Mula M, Pini S, Cassano GB: The role of anticonvulsant drugs in anxiety disorders: a critical review of the evidence. J Clin Psychopharmacol 2007, 27:263-272.
6. Feiner NF: Antiepileptic drug augmentation for treatment-resistant depression. J Clin Psychiatry 1997, 58:361-363.

7. Janati A, Shneker BF, Cios JS, Elliott JO: Suicidality, depression screening, and antiepileptic drugs: reaction to the FDA alert. Neurology 2009, 73:1710.

8. Wen X, Meador KJ, Loring DW, Eisenschenk S, Segal R, Hartzema AG: Is antiepileptic drug use related to depression and suicidal ideation among patients with epilepsy? Epilepsy Behav 2010, 19:494-500.

9. Thomas SP, Nandhra HS, Jayaraman A: Systematic review of lamotrigine augmentation of treatment resistant unipolar depression (TRD). J Ment Health 2010, 19:168-175.

10. American Psychiatric Association: Diagnostic and Statistical Manual for Mental Disorders. Fourth edition. Washington, DC: American Psychiatric Association; 1994.

11. Cheung $H$, Kamp D, Harris $E$ : An in vitro investigation of the action of lamotrigine on neuronal voltage-activated sodium channels. Epilepsy Res 1992, 13:107-112.

12. Lang DG, Wang CM, Cooper BR: Lamotrigine, phenytoin and carbamazepine interactions on the sodium current present in N4TG1 mouse neuroblastoma cells. J Pharmacol Exp Ther 1993, 266:829-835.

13. White HS: Mechanism of action of newer anticonvulsants. J Clin Psychiatry 2003, 64(Suppl 8):5-8.

14. Okada M, Kaneko S, Hirano T, Mizuno K, Kondo T, Otani K, Fukushima Y: Effects of zonisamide on dopaminergic system. Epilepsy Res 1995, 22:193-205. 
15. Okada M, Hirano T, Kawata Y, Murakami T, Wada K, Mizuno K, Kondo T, Kaneko S: Biphasic effects of zonisamide on serotonergic system in rat hippocampus. Epilepsy Res 1999, 34:187-197.

16. Sobieszek G, Borowicz KK, Kimber-Trojnar Z, Malek R, Piskorska B, Czuczwar SJ: Zonisamide: a new antiepileptic drug. Pol J Pharmacol 2003, 55:683-689.

17. Kinrys G, Vasconcelos e Sa D, Nery F: Adjunctive zonisamide for treatment refractory anxiety. Int J Clin Pract 2007, 61:1050-1053.

18. Murata M: Zonisamide: a new drug for Parkinson's disease. Drugs Today (Barc) 2010, 46:251-258.

19. Kaneko S, Okada M, Hirano T, Kondo T, Otani K, Fukushima Y: Carbamazepine and zonisamide increase extracellular dopamine and serotonin levels in vivo, and carbamazepine does not antagonize adenosine effect in vitro: mechanisms of blockade of seizure spread. Jpn J Psychiatry Neurol 1993, 47:371-373.

20. Hamilton M: A rating scale for depression. I Neurol Neurosurg Psychiatry 1960, 23:56-62.

21. Ventura J, Liberman RP, Green MF, Shaner A, Mintz J: Training and quality assurance with the Structured Clinical Interview for DSM-IV (SCID-I/P). Psychiatry Res 1998, 79:163-173.

22. Hamilton M: The assessment of anxiety states by rating. $\mathrm{Br} J$ Med Psychol 1959, 32:50-55.

23. Young RC, Biggs JT, Ziegler VE, Meyer DA: A rating scale for mania: reliability, validity and sensitivity. Br J Psychiatry 1978, 133:429-435.

24. McGahuey CA, Gelenberg AJ, Laukes CA, Moreno FA, Delgado PL, McKnight KM, Manber R: The Arizona Sexual Experience Scale (ASEX): reliability and validity. J Sex Marital Ther 2000, 26:25-40.

25. STAR*D Project: Sequenced Treatment Alternatives to Relieve Depression (STAR*D) project.[http://www.edc.pitt.edu/stard/public/assessment_forms. html].

26. Preskorn SH: Results of the STAR*D study: implications for clinicians and drug developers. J Psychiatr Pract 2009, 15:45-49.

\section{doi:10.1186/1744-859X-10-23}

Cite this article as: Fornaro et al:: An open pilot study of zonisamide augmentation in major depressive patients not responding to a low dose trial with duloxetine: preliminary results on tolerability and clinical effects. Annals of General Psychiatry 2011 10:23.

\section{Submit your next manuscript to BioMed Central and take full advantage of:}

- Convenient online submission

- Thorough peer review

- No space constraints or color figure charges

- Immediate publication on acceptance

- Inclusion in PubMed, CAS, Scopus and Google Scholar

- Research which is freely available for redistribution

Submit your manuscript at www.biomedcentral.com/submit
Biomed Central 